\title{
LE STATIF INDO-EUROPÉEN EN SLAVE
}

PAR

\author{
FREDERIK KORTLANDT
}

Dans un article classique (1933), N. Van Wijk a exprimé l'opinion que le nombre des parfaits transformés en présents baltiques et slaves est beaucoup plus grand qu'on ne le soupçonnait. Son hypothèse a été soutenue par Chr. Stang (1942) et A. Vaillant (1962, 1966), qui ont discuté le matériel plus en détail. On peut discerner les catégories suivantes.

Vx-sl. vědě « je sais » s'est transformé en présent athématique d'après les formes du pluriel, mais sur le thème du singulier à vocalisme - $o$ - caractéristique. La $3^{\mathrm{e}}$ personne du pluriel vědettŭ garde la désinence à degré zéro du parfait, à l'opposé de la désinence thématique de $s q t \breve{u}$ «ils sont » que l'on retrouve dans le participe vědqšt-.

$\mathrm{Vx}$-sl. mogQ « je peux » répond au prétérito-présent mag du germanique. Ce verbe s'est transformé en présent thématique à accentuation radicale. Vaillant a reconstruit une $2 \mathrm{e}$ personne du singulier athématique ${ }^{*}$ mo $\$$ i.

Vx-s1. gorěti «brûler », présent gori-, 3e pluriel gorętŭ, avec un participe présent gorq ̌̌t-, est du type de vědě, mais sans avoir gardé la flexion caractéristique, évidemment parce que la racine se termine par une consonne liquide. De même il faut reconnaître d'anciens parfaits dans polěti "flamber », bolěti « être malade », bojati se « craindre », stojati « être debout ». Correspondant à vědě mais contrastant avec mogQ, ces verbes ont des présents à accentuation désinentielle.

Le vocalisme radical -o- ne suffit pas pour établir un ancien parfait parce que l'origine de la voyelle peut être différente. Les verbes brati "lutter », klati «égorger», prati «fendre», bosti «piquer», kovati «forger», présents borje-, kolje-, porje-, bode-, kove-, représentent un type de présent intensif que Stang a identifié avec la formation védique de jánghanti, intensif de hánti «il frappe » (1942: 39-42). Dans le cas de xotět $i$ «vouloir », l'hypothèse d'un ancien parfait est gratuite. Il est probable que kosnqti (se) «toucher» a le vocalisme de l'intensif au regard du verbe lituanien kàsti « gratter» et du verbe slave česati «peigner», tandis que moknqti «être mouillé » peut remonter à un ancien parfait. En fait, il est séduisant de voir 
dans la différence accentuelle entre les verbes russes kosnút'sja et móknut' le résultat d'une origine différente.

Dans les verbes d'état en -ět $t$, présent - $i$-, à vocalisme radical réduit comme minnèti «penser », il est impossible de retrouver un parfait, parce que ce type ne peut être séparé du médio-passif védique mányate «il pense », grec maínomai « je suis furieux». De même il faut comparer bŭdèt $i$ 《veiller », pri-lĭpěti "être collé », kypět $i$ "bouillonner » aux correspondants védiques búdhyate "il est à l'éveil », lípyate "il est collé », kúpyati «il bouillonne ", latin cupiō « je désire». Ces verbes avaient une flexion de présent à suffixe apophonique $*_{-} e i-{ }_{-} *_{-} i$ - qui a laissé une trace en vieux prussien turei, turri « il a, ils ont " (Kortlandt 1987). Le participe *virq ̌̌t- du verbe virěti «bouillir » est suspect d'être analogique de gorqšt- (Vaillant 1966: 378).

En dehors des catégories qui viennent d'être énumérées il y a cinq verbes en -ět $i$, présent $-i$-, où le vocalisme radical fait difficulté pour l'identification historique de la flexion: běžati "fuir », letěti "voler", ležati "être couché », sěděti «être assis », velěti « vouloir, prescrire». La voyelle radicale de běžati et de sěděti en regard de celle de letěti, le žati et velěti s'explique par la loi de Winter (Kortlandt 1988). Les correspondants lituaniens bëgti, lếkti et vx-lit. pa-velti portent à croire que ces verbes avaient d'anciens présents athématiques. La flexion veli- de velěti révèle son origine d'optatif. L'élargissement dental de letěti invite à la comparaison avec l'itératif lituanien lakstýti, qu'on peut identifier avec l'itératif slave lètati comme aboutissement d'un présent balto-slave sg. *lekstä-, pl. *leksti- (Kortlandt 1989). Il en va de même pour le présent $b$ ěž et l'itératif běgati, qui peuvent recouvrir une flexion de présent plus ancienne sg. *bĕga-, pl. *běži- à côté du présent athématique reflété dans lit. bëgti.

Le cas des verbes d'état ležati et sěděti est différent. Je propose de dériver les présents leži- et $s e \check{d} i$ - du statif indo-européen, qui peut être défini comme une catégorie moyenne athématique à vocalisme radical - $e$-accentué. Le meilleur représentant de cette catégorie presque disparue déjà dans les langues les plus anciennes est le verbe védique sáye «je suis couché, il est couché », grec keĩmai, keĩtai. Je suppose que le paradigme balto-slave a de bonne heure adopté les désinences du parfait.

À partir de vx-sl. vědě « je sais » et goritŭ «il brûle » j'arrive à la reconstruction des désinences 1 re $\mathrm{sg}$. ${ }^{*}-a i$, 3 e $\mathrm{sg}$. - $e i$, où le ${ }^{*}-i$ final doit avoir été emprunté au présent athématique. Cet élargissement des désinences du parfait doit avoir été motivé par le besoin de faire une différence entre le présent et le prétérit nouveaux. C'est pourquoi il faut poser la question de savoir si l'on peut retrouver des traces du prétérit qui a fourni la motivation pour l'élargissement des désinences.

Une caractéristique remarquable qui distingue le slave des autres langues indo-européennes est la distribution complémentaire de l'aoriste sigmatique à accentuation désinentielle et de l'aoriste thématique à accentuation radicale qu'on trouve parmi les thèmes consonantiques (Dybo 1961). Cette distribution peut être de date balto-slave, parce que les correspondants lituaniens sont un prétérit en $-\dot{e}$ à accentuation mobile et un prétérit en $-o$ à accentuation radicale, respectivement. L'accentuation désinentielle de l'aoriste sigmatique s'explique 
par l'influence de l'aoriste radical, où la mobilité accentuelle doit avoir été normale.

Il est évident que les aoristes thématiques $i d$ - «aller », jad- «aller (en véhicule) » et krad- «dérober » représentent d'anciens imparfaits en vue des infinitifs $i t i$, jaxati, letton jât, krât. De même on peut supposer que les aoristes thématiques pad- «tomber », -lěz- «grimper» et vrŭg- «jeter » recouvrent d'anciens imparfaits, comme d'ailleurs les formes supplétives des $2^{\mathrm{e}}$ et $3^{\mathrm{e}}$ personnes du singulier du type vede dans le paradigme de l'aoriste sigmatique věs-de vesti «conduire». Je crois que la même chose vaut pour l'aoriste thématique - běg-de běgnqti «fuir », lit. bëgti. Il est à noter que $i d$-, jad-, pad-, -lěz-, -bĕg-sont tous des verbes de mouvement.

Dans ce contexte il est probable que les aoristes thématiques leg-et $s$ ědreprésentent d'anciens imparfaits qu'on peut identifier aux anciens prétérits de leži- et sědi-. Le développement peut être comparé à celui de l'anglais, où la forme de parfait sat signifie non seulement «était assis » mais aussi «s'est assis ». Ce développement est beaucoup plus probable que la création d'un présent en $-i$ - à partir d'un ancien aoriste thématique, pour laquelle on voit mal la motivation. En effet, il y a un parallèle significatif dans l'aoriste thématique mog-, qui est issu d'un paradigme de parfait et a fourni la base pour la création d'un présent thématique. L'accentuation radicale de ce paradigme porte à croire que la forme reconstruite de la $2^{\mathrm{e}}$ personne du singulier * moši n'est pas ancienne.

L'hypothèse que l'aoriste thématique leg-, sěd- et le présent leži-, sědireprésentent en fin de compte un seul paradigme de statif original explique la coexistence de l'accentuation radicale de l'aoriste et désinentielle du présent d'une même racine, chose inattendue en slave. Un autre aoriste thématique qui à cause du vocalisme radical peut être soupçonné de refléter un ancien statif est - ̌̌ez- de čeznqti « défaillir », qu'on peut comparer avec lit. kèžti « faiblir ». Les verbes $o b$-rěsti «trouver » et $s \breve{u}$-rěsti «rencontrer » allient un présent à infixe nasal avec l'aoriste thématique -rět-, qu'on ne saurait séparer du thème grec heurē-, quoique les détails du développement restent obscurs.

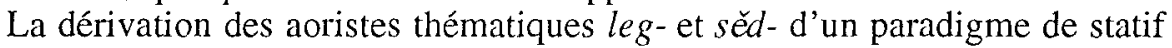
incite à reconsidérer les formes correspondantes du grec et du védique. En grec homérique la forme lékto joue le rôle d'un aoriste, mais « il est probable que nous avons affaire en réalité à un ancien présent athématique " (Chantraine 1973 : 384), c'est-à-dire à un paradigme rappelant le statif originel. L'aoriste thématique védique sáda- a la forme et l'accentuation d'un imparfait actif. Il est possible que ce paradigme remplace une formation athématique plus ancienne.

La difficulté à laquelle se heurte l'hypothèse que leg-et sěd-représentent des statifs indo-européens consiste dans l'existence des statifs indubitables véd. śáye, áste, gr. keĩtai, hẽstai pour «être couché » et « être assis ». Les formations dérivées des racines *legh-et *sed-dans les langues indo-européennes indiquent plutôt une signification dynamique. C'est pourquoi je crois qu'il faut partir de présents athématiques actifs *leghmi, *sedmi, formation reflétée dans l'aoriste védique sáda-, et que ceux-ci ont fourni la base pour la création de statifs *legh-, *sed-à désinences moyennes. Ces statifs ont remplacé les correspondants de keĩtai et de héstai en balto-slave et en germanique (Kortlandt 
1990 : 8), ce qui suggère la possibilité d'un développement dialectal indoeuropéen. En balto-slave, ils ont de bonne heure adopté les désinences du parfait, dont ils ont partagé le développement ultérieur. Ce n'est que le vocalisme radical qui trahit leur origine de statif.

(Université de Leyde)

\section{BIBLIOGRAPHIE}

P. Chantraine, 1973, Grammaire homérique, t. I : Phonétique et morphologie, Paris.

V. A. DYво, 1961, «Ударение славянского глагола х формы старославянского аориста », Краткие сообщения Института славяноведения, 30, p. 33-38.

Fr. KORTLANDT, 1987, «The formation of the Old Prussian present tense », Baltistica, 23/2, p. $104-111$.

ID., 1988, «Remarks on Winter's law », Studies in Slavic and general linguistics, 11, p. 387-396.

ID., 1989, «Lithuanian statýti and related formations », Baltistica, 25/2, p. 104-112.

ID., 1990, «The Germanic third class of weak verbs », North-Western European language evolution, 15, p. 3-10.

Chr. S. STANG, 1942, Das slavische und baltische Verbum, Oslo.

A. VAIllaNT, 1962, «Le parfait indo-européen en balto-slave », Bulletin de la Société de linguistique de Paris, 57, p. 52-56.

ID., 1966, Grammaire comparée des langues slaves, t. III : Le verbe, Paris.

N. VAN WuK, 1933, «Le problème des prétérito-présents slaves et baltiques », Studi baltici, 3, p. 134-139. 